\title{
Experimental investigations of the steady flow through an idealized model of a femoral artery bypass
}

\author{
Corina Giurgea $^{1}$, Florin Bode ${ }^{1, a}$, Octavian Ioan Budiu ${ }^{2}$, Lucian Nascutiu ${ }^{1}$, Daniel Banyai ${ }^{1}$ and Mihai Damian ${ }^{3}$ \\ ${ }^{1}$ Technical University of Cluj-Napoca, Faculty of Mechanics, Dept.of Mechanical Engineering, 400103, Romania \\ ${ }^{2}$ Iuliu Hatieganu University of Medicine and Pharmacy Cluj-Napoca, Surgical Clinic II, 400006, Romania \\ ${ }^{3}$ Technical University of Cluj-Napoca, Faculty of Machine Building, Dept. of Manufacturing Engineering, 400103, \\ Romania
}

\begin{abstract}
The present paper presents the steps taken by the authors in the first stage of an experimental program within a larger national research project whose objective is to characterize the flow through a femoral artery bypass with a view to finding solutions for its optimization. The objective of the stage is to investigate by means of the PIV method the stationary flow through a bypass model with an idealized geometry. A bypass assembly which reunites the idealized geometry models of the proximal and distal anastomoses, and which respects the lengths of a femoral artery bypass was constructed on the basis of data for a real patient provided by medical investigations. With the aim of testing the model and the established experimental set-up with regard to their suitability for the assessment of the velocity field associated to the steady flow through the bypass, three zones that can restore the whole distal anastomosis were PIV investigated. The measurements were taken in the conditions of maintained inflow at the bypass entry of $0.91 / \mathrm{min}(\operatorname{Re}=600)$. The article presents comparatively the flow spectra and the velocity fields for each zone obtained in two situations: with the femoral artery completely occluded and completely open.
\end{abstract}

\section{Introduction}

Nowadays, vascular reconstructions represent the main part of vascular surgeon work and are usually done to correct the effects of atherosclerosis, which may affect almost any vessel in the body.

At the lower extremity level, atherosclerosis produces peripheral artery disease (PAD), of which one clinical form is critical limb ischemia. According to [1] the treatment of this disease involves femorodistal arterial reconstruction in over $60 \%$ of cases and almost 20000 of this kind of surgery take place annually only in the UK.

The femorodistal arterial reconstruction referred also as femoral artery bypass graft (hereinafter named as FABG) consists in bypassing the stenosed (narrowed) or occluded (blocked) zone(s) of the femoral artery with a graft. The graft is usually the great saphenous vein harvested from the patient, or a prosthesis (a tube made of synthetic materials: PTFE, Dacron).

The connections between the graft and the artery are called anastomoses. The distal anastomosis is located downstream of the stenosis and corresponds to the zone where the graft is reconnected (by suturing) to the native artery after they have been branched out at the proximal anastomosis, situated upstream of the stenosis.

The most used procedure in this kind of surgery is the construction of an end-to-side bypass graft. With regard to a femoral artery bypass, this term is used to indicate that the graft ends are sutured laterally to the native femoral artery by means of a longitudinal incision.

Despite the technical success of the surgery, restenosis and late graft failure often appear within 2-24 months after operation. One of the primary causes of the long term graft failure is a phenomenon called intimal hyperplasia $(\mathrm{IH})$, a process in which the inner and middle layer of the blood vessel wall increase in thickness leading to graft occlusion and failure.

Since clinical studies had suggested that aspects related to the blood flow within the distal anastomoses might be one of the causes of IH [2], a great number of interdisciplinary studies have been undertaken in order to identify and quantify the hemodynamics aspects that could be responsible for the $\mathrm{IH}$ occurrence and development [3-4]. A comprehensive review of the numerical but also the in vivo and in vitro experimental studies that were conducted with the above mentioned aim during almost 20 years can be found in [5].

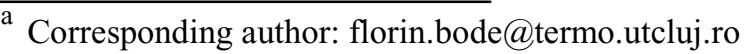


As a result of the numerous studies cited above, a consensus has been reached with regard to the fact that the ,disturbed flow” (a term that encapsulates flows with stagnation points, separation/recirculation zones, vortices and secondary flows), as well as hemodynamics parameters like the wall shear stress (WSS), the spatial wall shear stress gradients (SWSSG) or oscillating shear (OSI) are important factors in IH.

In the last ten years most studies have had the objective of quantifying the influence that the above mentioned factors have on the mechanisms that trigger the occurrence of $\mathrm{IH}$ in anastomoses with different configurations similar to those encountered in real patients [6].

Despite the advances in the field of medical investigations techniques (particularly that of medical imaging like MRI or CT), the in vivo assessment of certain hemodynamics parameters (e.g. WSS, SWSSG) is still either impossible or difficult/unreliable. In this context, the continuous progress in computerized image processing and in computational fluid mechanics (CFD) are turning numerical simulation, with its demonstrated versatility, into the most appropriate tool in quantifying the local hemodynamics in anastomoses with real configurations [7].

As the numerical results should be validated, there have been carried out some in vitro investigations of the flow through different anastomosis configurations. These investigations were based on the use of optical methods, mostly on PIV (Particle Image Velocimetry), in order to map the velocity field to the entire anastomosis. But as stated in [8], quantifying parameters like SWSSG or even WSS by experimental approaches including PIV „remains a formidable task". This statement is justified if one takes into account the fact that the magnitude of WSS is proportional to the velocity gradient near the wall and that accurate measurements of the velocities close to the wall, especially when it is curved, is one of the most challenging task in PIV investigations. Some particular aspects of this kind of measurement, such as avoiding the distortion and the refraction at the walls, results in special conditions that the anastomosis models and the experimental setup should comply with [9].

Most numerical simulations and PIV investigations carried out in the last years regarded to the role of hemodynamics in IH occurence in coronary artery bypass graft $(\mathrm{CABG})$. It would be difficult to extrapolate their results to the case of the femoral artery bypass even if some similarities concerning the anastomoses' configurations could be found [4]. One can take into account that not only the dimensions (especially the graft length which in $\mathrm{CABG}$ is smaller than in femoral artery bypass graft) are different but also the flow conditions.

As noted by [10], research regarding the influence of fluid mechanics factors in the occurrence of $\mathrm{IH}$ has concentrated, with very few exceptions, only on investigating distal anastomosis because this would be, according to clinical studies, the predilect location for IH occurrence. But features of proximal anastomosis hemodynamics might trigger the initiation of $\mathrm{IH}$. Moreover, Lee et al, cited by [10] reported that the flow through proximal anastomosis has an influence on the distal anastomosis site specific flow condition

Another observation gleaned from observing the literature devoted to the topic is that models used are in many cases idealized geometry models (they allow for a symmetry plane), but this was arbitrary established.

Both observations above are valid also for PIV studies that regard the flow through a distal anastomosis of a FABG [11-13].

In this context, a national interdisciplinary research project was initiated, to which the authors of the present paper also took part. A major objective of the project is to constitute a detailed and rigorous analysis of the flow through a FABG. The geometric models that will be used to realize both the numerical models for numerical simulation and technical models for the PIV investigations are built starting from the morphometric and functional data of real patients. They will include both proximal and distal anastomosis, and will take into account the real dimensions of the bypass.

The present article only treats aspects regarding the experimental program of the project's first stage, whose objective is to investigate, by the PIV method, the stationary flow through a bypass with idealized geometry. With this aim an experimental facility was built. It will be described in the section 2 after a briefly presentation of the way the authors established the idealized geometry of the bypass and reproduced it in a technical model suitable for PIV investigations. The experimental investigation reported here was intended to test the way in which the bypass assembly model built in the project and the experimental setup allow us to assess the velocity fields in the distal anastomosis. The third section of the paper presents the results obtained for two test case scenario as well as the observations that can be made with regard to these results.

\section{Materials and method}

\subsection{Experimental set-up}

\subsubsection{The optically transparent models of the anastomoses}

A model of a bypass with idealized geometry should be seen as a model in which both artery and graft are axisymmetric, and their branching/reattaching plane is considered in the symmetry plane of the model.

In our project, the input information for the construction of the bypass assembly model was the morphometric data that came out from the two-weeks postoperative EcoDoppler and gandolinum enhanced AngioMRI investigations of a real patient. The patient underwent an end to side femoral artery bypass with a Dacron prosthesis that was placed between the two anastomoses in a manner resembling an $\mathrm{S}$ shape (circling like a spiral) around the obstructed femoral artery. This arrangement has been enforced by the patient anatomy.

As the authors' purpose for this stage of the project was that of creating a model with an idealized geometry, the 2D geometry was established by calculating angles and length of the projections on the symmetry plane of all the out of plane curvature. The calculus was done by using the data measured on the DICOM images of the 
projections of the bypass on two perpendicular planes (sagital and frontal). The median plane was set as the symmetry plane. The DICOM images were provided by an General Electric 1 Tesla Angio MRI.

As agreed with the surgeons and with the partners in charge with the CFD part of the project, and based on the results of the above mentioned calculation, the authors established the geometry of the complete bypass. This geometry will be used to build both numerical and technical models.

Thus, the femoral artery is modeled in the shape of a straight tube with a length of $455.3 \mathrm{~mm}$ (considered between the proximal and the distal anastomoses) and a diameter of $8 \mathrm{~mm}$. To ensure the stability of the flow in the entry section and the optical access to the distal artery outlet, two supplementary segments were added at the entrance of the proximal anastomosis and at the outlet of the distal anastomoses. The graft is of the same diameter as the host vessel. The graft arms follow the profile of Bezier curves, the distance between graft and native artery, and the value of the anastomosis angle being 27.8 $\mathrm{mm}$ and $39.3^{\circ}$ respectively, in the area of the proximal anastomosis, and $21.6 \mathrm{~mm}$ and $23.3^{\circ}$ in the area of the distal anastomosis [14].

A digital model of the bypass was created using CAD packages SolidWorks and Catia V5R20. By reason of manufacturing technology the complete bypass digital model was divided into three blocks. Two of them corresponding to the proximal and distal anastomoses where used in manufacturing the optically transparent models of the anastomoses.

We should mention here that in conformity to [9], besides complying with the conditions imposed by ensuring geometrical similitude, the models suitable for PIV measurements must comply with two specific conditions. Thus, the material of which the model is made must be optically transparent and have a refraction index that can be easily matched to that of the working fluid. This last constraint is imposed in order to eliminate as much as possible the reflections and the optical distortions that arise at the interface between the fluid and the solid walls especially when these ones are curved.

One can find in the literature, examples (or even lists) of solid optically transparent materials and liquids of appropriate refraction indices [15]. However, achieving refractive-index-matched phantoms is difficult, because of limitations imposed by the fabrication technologies, (especially when flow configurations with complex surfaces must be fabricated) and by the corrosive and/or dangerous actions of some of the possible working fluids.

The authors developed during this stage of the project a manufacturing technology of models consisting of idealized bifurcations and having a high degree of transparency. Following the digital model construction, the branch channels were CNC milled (on a DMU 50/5 Axis CNC Universal Milling Machine Tool) in blocks of Plexiglas, with a high degree of transparency.

A Plexiglas with a refractive index $n=1.491$ at the temperature $\mathrm{T}=20^{\circ} \mathrm{C}$ was used in order to manufacture the anastomoses' models presented in figure 1.
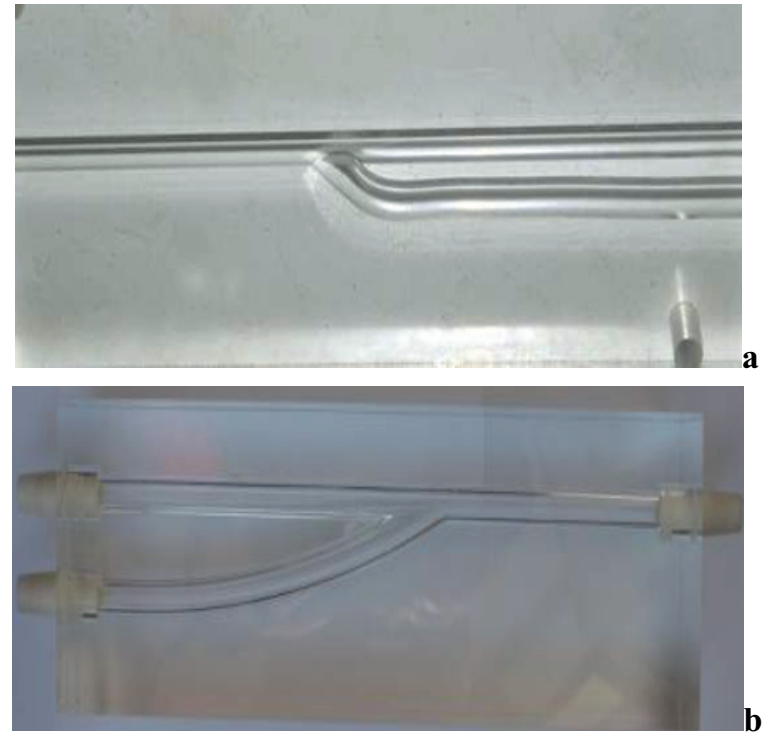

Figure 1. The optically transparent models with idealised geometry of the proximal (a) and distal (b) anastomoses

Figure 2 presents the bypass assembly as it was modeled with two blocks representing the anastomoses connected by two Tygon Lab tubings with an inner diameter of $5 / 16 "$ and outer diameter of $7 / 16$ " that modeled the graft and the femoral artery. A manual pinch valve (Cole \& Parmer model TW 98002-20) that fits on tubing with the outer diameter of $7 / 16$ " was used to simulate the stenosis.

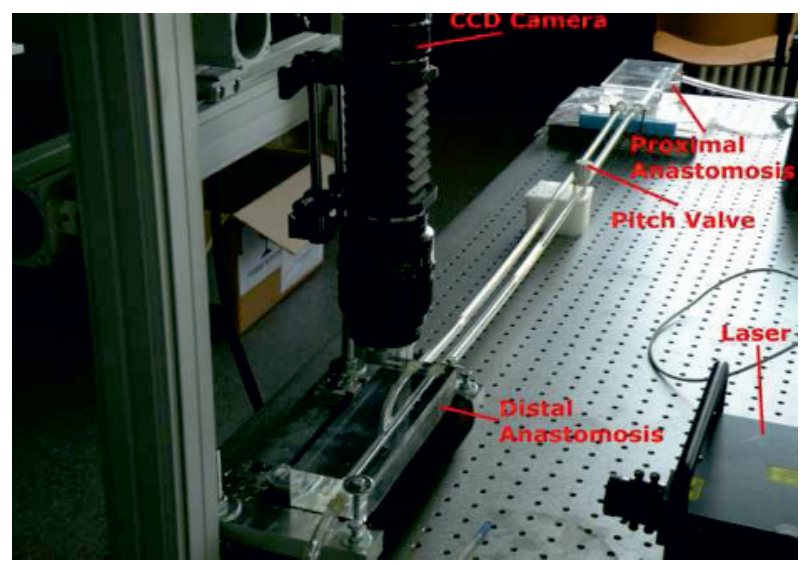

Figure 2. The complete bypass model

\subsubsection{The flow circuit}

The experimental setup used for the PIV investigations is shown in figure 3 . The experimental facility integrates a flow circuit and a 2D PIV system.

In the flow circuit the steady flow is generated by the Gear Pump System with Benchtop Digital drive, 0.91 $\mathrm{ml} / \mathrm{rev}$ (model 75211-30/-35, Cole-Parmer) (number 1 in figure 3 ). The system is provided with a Realtime display of the flowrate value.

Tygon tubings with an inner diameter of 5/16" and outer diameter of $7 / 16$ "were used for easy connection of the pump system with the reservoir (number 2 in figure 1) and the complete bypass model including the proximal and distal anastomoses (number 3 and 6 in figure 1) and the pinch valve (number 5 in figure 1 ). 
A HemoLab Ultrasound Flow Meter system (number 10 in figure1) provided with Realtime display of the flowrate value was used to monitorise the flowrate through the graft. The clamp-on sensor (number 4 in figure 1) that fits onto a standard tubing of size 5/16" inner diameter and wall thickness of $3 / 32$ " was mounted on the Tygon tubing that simulates the graft of the bypass, in compliance with the conditions imposed by the flowrate ultrasonic measurement method.

The working fluid was a solution of glycerol-towater ( $37: 61$ by volume) whose refractive index was measured in the temperature conditions of the experimental facility $\left(\mathrm{T}=19.6{ }^{\circ} \mathrm{C}\right)$ with a Digital Laboratory Refractometer ( model 300034 Sper Scientific Ltd). The average value of the refractive index was found to be $n=1.38$. The viscosity of the fluid was also measured in the temperature conditions of the experimental facility with a Tuning Fork Vibration Viscometer (model SV 10 A\&D Japan). The average value of the viscosity was 4.12 cPoise (at $\mathrm{T}=19.6{ }^{\circ} \mathrm{C}$ ).

With the aim to accurately measure the velocities near the wall, the working fluid was seeded with Red fluorescent $7 \mu \mathrm{m}$ diameter dry particle (ThermoScientific USA) with a specific gravity of $1.055 \mathrm{~kg} / \mathrm{m}^{3}$, a refractive index $\mathrm{n}=1.59$ at $589 \mathrm{~nm}$ and an excitation/emission wavelengths of $542 / 612 \mathrm{~nm}$.

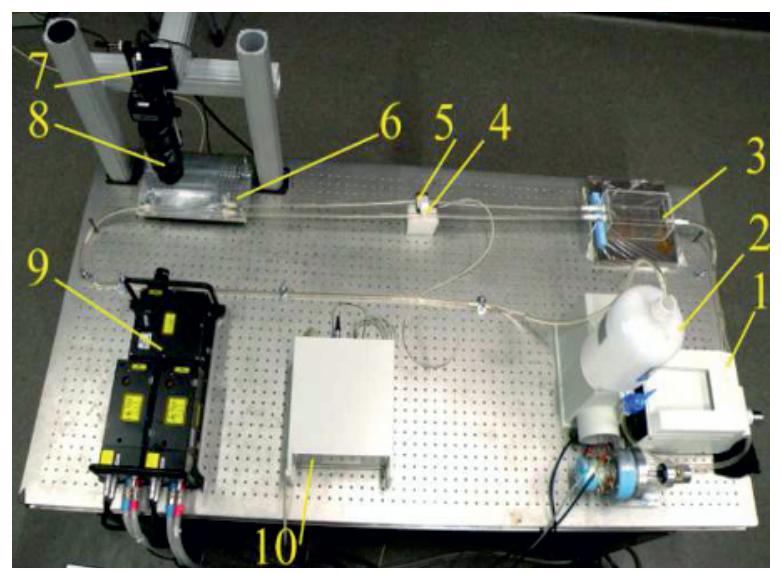

Figure 3. The experimental set-up used for PIV investigations

\subsubsection{The 2D PIV system}

The flow in the horizontal plane passing through the test section symmetry plane was investigated using the TSI 2D PIV System (TSI Incorporated, USA).

The illumination of the flow field is assured by a double pulse laser head $\mathrm{Nd}$ :Yag (number 9 in figure 3) that provides two $532 \mathrm{~nm}$ green light pulses, each one with a duration of $10 \mathrm{~ns}$ and an energy of $120 \mathrm{~mJ}$. Each laser beam is expanded into a light sheet with a $1 \mathrm{~mm}$ thick waist by means of the light sheet optics. In the experiments presented here, the light sheet optics consisted of a $200 \mathrm{~mm}$ focal length spherical lens and a -25 focal length cylindrical lens.

The Laser Pulse Synchronizer of the system provides all the control and activation signals including those for precision frame straddling. The synchronizer is programmed through the INSIGHT 3G software running on a Dell Workstation (Intel Dual Core Xenon 5150).
The images of the particles are recorded with the Powerview $17 \mathrm{CCD}$ camera (number 8 in figure 3 ) that has: 4 MP $(2048 \times 2048$ pixels $)$ resolution, an image capture rate of 16 frames / second and the ability to record sequential images in separate frames. The camera is placed above the model and perpendicular to the flow field. It was mounted on a Manual 2 " (50 $\mathrm{mm}$ ) linear translation stage (number 7 in figure 3 ) whose fine adjustment screw provides a displacement of $0.25 \mathrm{~mm} / \mathrm{rev}$. Thus, the camera could be finely displaced in order to capture the images from different zones of the anastomosis without moving the model.

The camera was equipped with the Micro-Nikkor $105 \mathrm{~mm} \mathrm{f} / 2.8$ lens (Nikon, Japan). In order to focus better, a Nikkor PK 13Macro extensions ring and a Macro bellows extension for Nikon AR-11 were supplementary mounted. Thus, the DOF (depth of field) was $17 \mathrm{~mm} \times 17 \mathrm{~mm}$. A low pass filter (Optical Filter $545 \mathrm{~nm}$ Fluro) was installed on the camera to cut off reflections and pass only the "orangelight" scattered by the fluorescent particles.

\subsection{Experimental procedure and PIV data processing}

With the aim to map the velocity field through the entire distal anastomosis, this was divided into the three zones of interest that can be seen in figure 4 a. Zone 1 corresponds to the entrance in the native artery of the flow coming out from the graft, zone 2 contains the heel of the anstomosis and the so-called proximal artery outlet, while zone 3 contains the so-called distal artery and the toe of the anastomosis (see figure $4 \mathrm{~b}$ ) The borders of the zones overlapped slightly, so as not to miss any information.
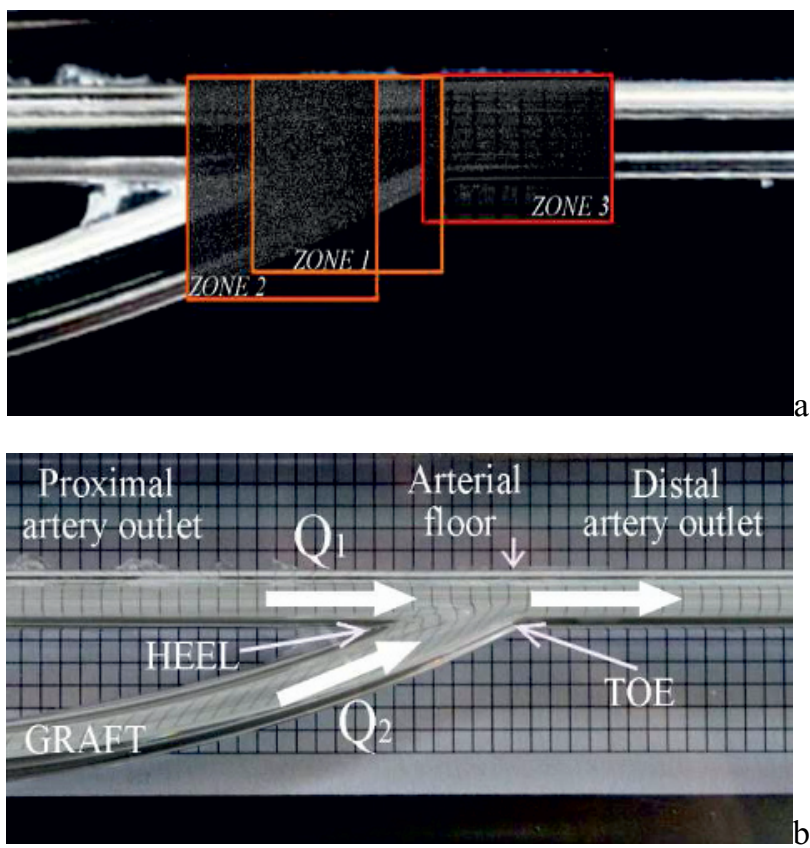

Figure 4. The distal anastomoses as it was divided into the three zones of interests for PIV investigations (a). Branching flowrates in distal anastomoses and different regions of an anastomosis as they are conventionnaly termed (b). 
A constant inflow rate of $0.91 /$ min was assured at the entrance of the proximal artery for all the measurements carried out in conformity with two test case scenario: test case A that simulates the steady flow through a blocked artery (100\% stenosis degree or $\mathrm{Q}_{1}=0$, in conformity to figure $4 \mathrm{~b}$ ) and test case $\mathrm{B}$ that simulates the steady flow through a completely open femoral artery (or $0 \%$ stenosis degree).

As the flow aspects (spectra and velocity magnitudes) within the three zones are expected to be different, the separation time $(\Delta \mathrm{t})$ between two images recorded by the camera was set to different values: from $100 \mathrm{~ms}$ (for the entrance in the native artery) to $200 \mathrm{~ms}$ (for the heel of the anastomosis zone).

As a preprocessing technique, the background subtraction was used to create a mask of the non-flow region.

The ensemble PIV algorithm was used on 500 pairs of images. As grid engine, the RecursiveNyquistGrid was used with a starting spot dimension of $64 \times 64$ and a final spot dimension of $32 \times 32$. Zero Pad Mask was used as Spot Mask Engine, FFT Correlation Engine and Gaussian Peak as Peak Engine.

\section{Results and discussions}

The results obtained after processing the PIV images are presented in figures 5-7. In each of this figure the left column corresponds to the results obtained for the test case $\mathrm{A}$ and the right column corresponds to the results obtained for the test case B.

For each of the two test cases, the spectra and the velocity field corresponding to the steady flow within the entire distal anastomosis of the bypass assembly could be reconstructed from the spectra, respectively the velocity fields, corresponding to each of the zones of interest.

Even without such a reconstruction exercise, a simple comparison of the streamtraces corresponding to each zone of interest for the test case A (images a in figures 57) to that corresponding to the same zone but for the scenario $B$ (images $b$ in figures 5-7) makes evident the completely different flow structures in the two cases. The flow structure in case A comprises a stagnation point on the arterial floor and a vortex that overshot the heel of the anastomosis and extended inside the so called proximal artery outlet (see figures 5-6 a). The stagnation point is formed when the flow from the graft is deviated at the entrance of the anastomosis and is bifurcated into the deflected main flow through the distal artery outlet and into the recirculated flow (the vortex) through the proximal artery outlet.

A careful examination of the streamtraces corresponding to the deflected main flow (see figure 7 a) allows us to notice their tendency to converge. This tendency is more detectable starting with the cross section considered at $\mathrm{x}=8 \mathrm{~mm}$.

By examining the velocity vectors depicted in figure $7 \mathrm{c}$ one can notice that their crosssectional distribution is once again skewed, starting with the cross section considered at $\mathrm{x}=8 \mathrm{~mm}$. Based on this observation we might advance the idea that the curved streamtraces and the double skewed distribution of the velocity vectors indicate a secondary flow that could not been assessed by 2D PIV investigation.

One of the very few studies that we can refer to in order to "validate", even only qualitatively, the results obtained is the one undertaken by Hughes and How [16]. Their visualizations of stationary flows in a distal anastomosis model with a $30^{\circ}$ angle, and the same diameter of $8 \mathrm{~mm}$ for the graft as well as for the proximal artery that was fully occluded evidenced for $\mathrm{Re}=600$ the same structure of the flow as the one encountered in our investigations. Furthermore, their visualizations made possible the observation of path lines curving already at $\mathrm{Re}=300$, indicating the presence of a secondary flow. This is still more obvious at $\mathrm{Re}=600$, and at $\mathrm{Re}=850$ even the main flow separation at the toe of the anastomosis was observed.

The flow structure in case B does not present the same elements of a "disturbed flow" as observed in case A. Still, a shearing zone can be identified appearing already at the confluence of the two streams.

All the images in which velocity distributions appear, with or without superposed vectors, evidence the fact that measuring velocities near the artery and graft walls was not possible.

Figure 7 shows that for zone 3 corresponding to the distal anastomosis outlet, it was not possible to measure velocities for an area $0.5 \mathrm{~mm}$ from the near wall and 0.75 $\mathrm{mm}$ from the outer wall, which corresponds to $6 \%$ and 9 $\%$ respectively of the diameter. The observed difference is explained by the fact that the laser sheet penetrates the model through the near wall and exits through the outer wall, its intensity being attenuating by the passing through the Plexiglas walls and the working fluid. It must be noted that we do not have the certainty that the laser sheet was perfectly superimposed over the symmetry plane of the anastomoses.

The failure to determine the velocity fields in the immediate proximity of the anastomosis walls can be attributed to the large difference between the refraction indices of the model and of the working fluid.

If for the refractive index of the work fluid measurements were taking during the whole course of the investigation in the temperature conditions of the experimental facility, the same was not possible for the model itself. We can venture the idea that the catalogue value of the refractive index for the model's Plexiglas can no longer be considered valid, due to the modifications induced by tensions occurring during its processing by chipping.

Given that, due to economic and availability reasons, the construction technology of the model cannot be changed; a solution would be the change of the working fluid.

A solution of water, glycerol and NaI, whose refractive index could be adjusted by changing in $\mathrm{NaI}$ concentration, could be a candidate. From our earlier experience with this working fluid we know that it gets yellow quickly and this gives rise to the yellowing of the model. According to a recent paper [17] this problem could be overcome by easily dissolving $0.1 \%$ by weight of sodium thiosulphate. 

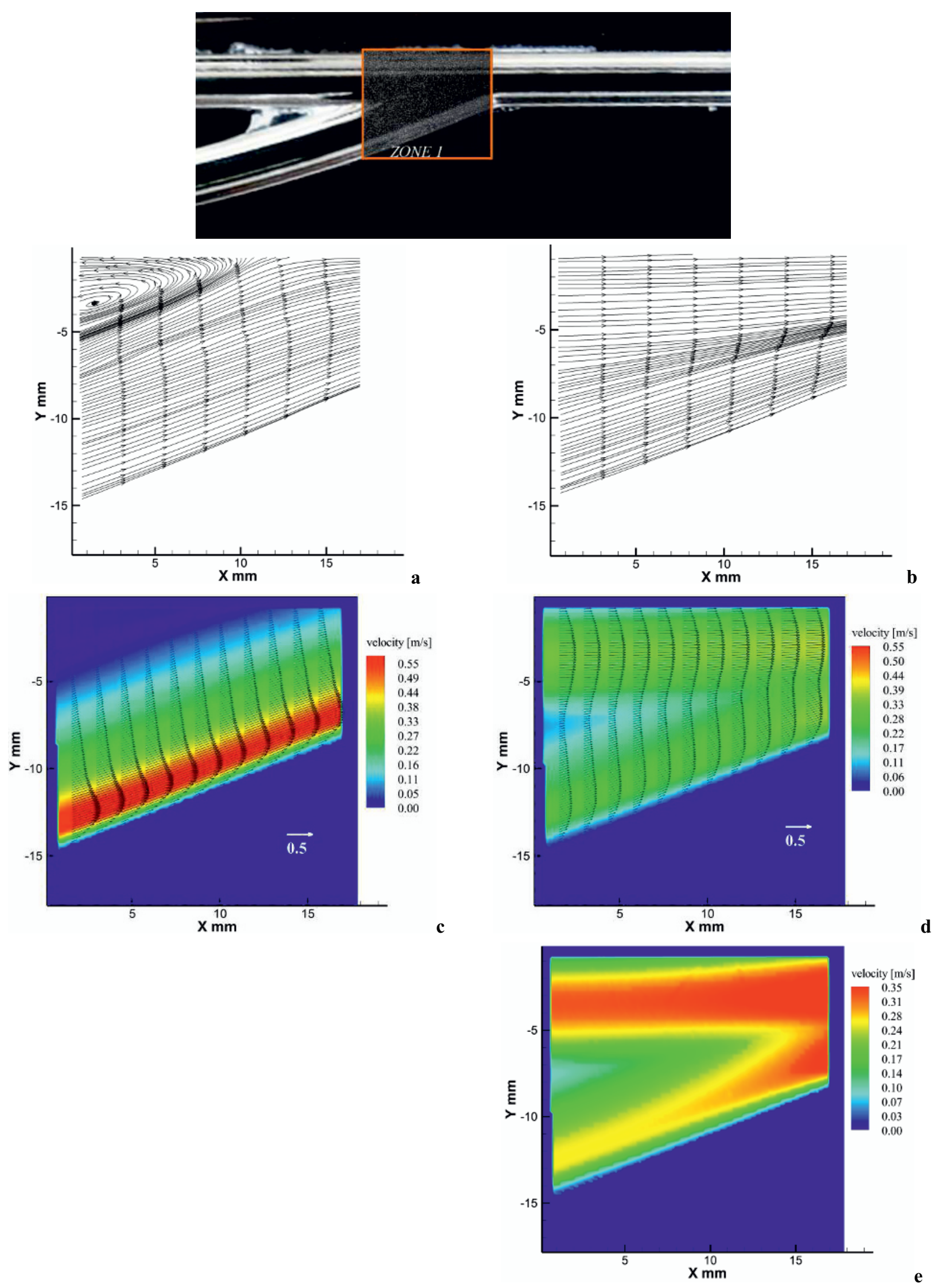

Figure 5. The PIV investigation results for Zone 1. Streamtraces for $100 \%$ stenosis (a) and $0 \%$ stenosis (b); Velocity vectors superimposed on velocity contours for $100 \%$ stenosis (c) and $0 \%$ stenosis (d); Velocity contours for $0 \%$ stenosis (close-up velocity scale)(e) 

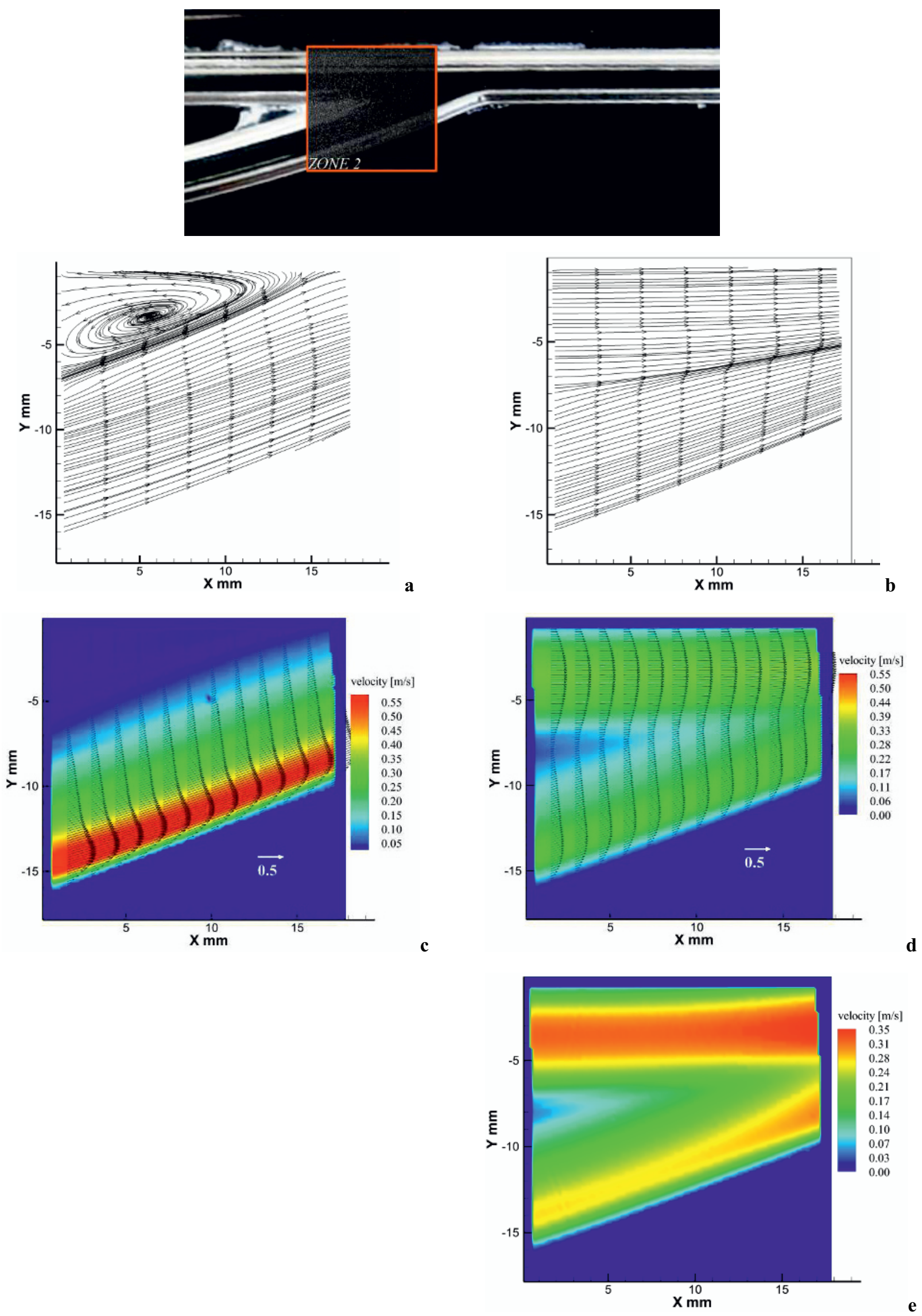

Figure 6. The PIV investigation results for Zone 2. Streamtraces for $100 \%$ stenosis (a) and $0 \%$ stenosis (b); Velocity vectors superimposed on velocity contours for $100 \%$ stenosis (c) and $0 \%$ stenosis (d). Velocity contours for $0 \%$ stenosis at a close-up velocity scale (e) 

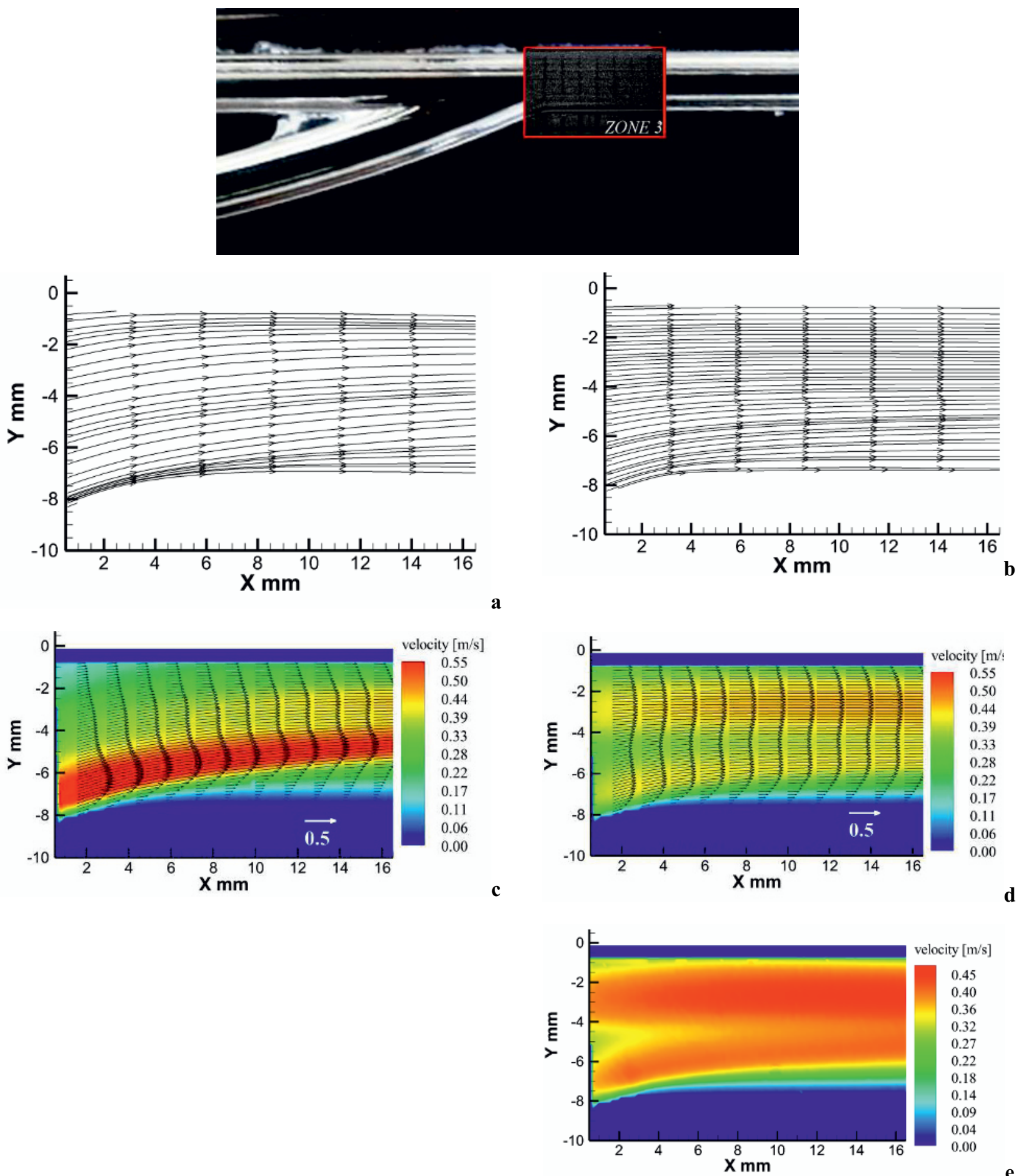

Figure 7. The PIV investigation results for Zone 3. Streamtraces for $100 \%$ stenosis (a) and $0 \%$ stenosis (b); Velocity vectors superimposed on velocity contours for $100 \%$ stenosis (c) and $0 \%$ stenosis (d). Velocity contours for $0 \%$ stenosis at a close-up velocity scale (e) 


\section{Conclusions and future work}

The PIV investigations devoted to testing the bypass model and the setup have shown that determining the velocity field in the immediate proximity of the walls was not possible with the current setup. A possible cause could be the non-compliance with the condition of compatibility of the refractive indices of the model and the working fluid. In trying to improve the quality of the PIV images, changing the work fluid into a solution of water, glycerol, $\mathrm{NaI}$ and $0.1 \%$ by weight of sodium thiosulphate will be attempted.

However, we succeed in mapping the velocity field of the steady flow through the entire distal anastomosis, excepting the immediate proximity of the walls. Thus, the flow structure in two extreme cases corresponding to a blocked respectively fully opened artery could be assessed.

The results of our measurements for the case that simulates the flow through the bypass with a blocked femoral artery evidenced the "disturbed flow" structure of this flow. This flow "encapsulates" a stagnation point on the arterial floor, a vortex that overshot the heel and extended in the proximal artery outlet and even a secondary flow whose occurrence conditions seems to be present in the distal artery outlet. This structure was in concordance with that evidenced by a comprehensive visualization study [17].

\section{Acknowledgement}

This work was supported by the grants of the Romanian National Authority for Scientific Research, CNCS UEFISCDI, project number PN-II-PT-PCCA-2011-3.20512 and CNMP 82086 MAACH.

\section{References}

1 R.K. Fisher, T.W. How, I.M. Toonder, M.T.C. Hoedt, J.A. Brennan, G.L. Gilling-Smith, P.L. Harris, Eur J Vasc Endovasc Surg 21, 520-528 (2001)

2 H.S. Bassiouny, S. White, S. Glagov, E. Choi, D.P Giddens, C.K. Zarins, J Vasc Surg 15, 708-717 (1992)

3 C. Kleinstreuer, J.R. Buchanan, M. Lei, G.A. Truskey, in: Leondes C., Cardiovascular Techniques. Biomechanical Systems, Techniques and Applications, CRC Press, London, II, 1-69 (2001)

4 F. Migliavacca, G. Dubini, Biomechan Model Mechanobiol 3, 235-250 (2005)

5 H. Haruguchi, S. Teraoka, J Artif Organs 6, 227-235 (2003)

6 T. O Brien, M. Walsh, T. McGloughin, Annals of Biomedical Engineering Vol.33, No. 3, 310-322 (2005)

7 D.A. Steinman, Annals of Biomedical Engineering Vol 30, 483-497 (2002)
8 F.L. Xiong, C.K. Chong, J Biomech 40, 2872-2881 (2007)

9 P.H. Geoghegan, N.A. Buchmann, C.J.T. Spence, S.Moore, M. Jermy, Exp Fluids 52, 1331-1347 (2012)

10 L P Chua, W-F Ji, C M Yu, T-M Zhou, Y S Tan, Proc I Mech E Vol 122 Part H: J. Engineering in Medicine 249-263, (2008)

11 M. Heise, U. Kruger, R. Ruckert, R. Pfitzman, P. Neuhaus, U. Settmavher, Eur J Vasc Endovasc Surg 26, 357-366 (2003)

12 M. Heise, S. Schmidt, U. Kruger, R. Ruckert, S. Rosler, P. Neuhaus, U. Settmacher, J Biomech 37, 1043-1051 (2004)

13 J.D. Gray, I. Owen, M.P. Escudier, Exp Fluids 43, 535-546 (2007)

14 S. Danaila, Scientific Research Report for the Project CNMP 82086 MAACH, 2011 (in romanian)

15 T.T. Nguyen, Y. Biadillah, R. Mongrain, J. Brunette, J-C. Tardif, O.F. Bertrand, J Biomech Eng 126, 529-535 (2004)

16 P.E. Hughes, T.V. How, J. Biomechanics Vol.29, No. 7, 855-872 (1996)

17 M.Y. Yousif, D.W. Holdsworth, T.L. Poepping, Exp Fluids 50, 769-774 (2011) 